\title{
Práticas de educação em saúde para o público idoso: perspectivas e reflexões
}

Health education practices for the elderly public: perspectives and reflections

\author{
Prácticas de educación para la salud para el público mayor: perspectivas y reflexiones
}

Talita Mendonça Sales ${ }^{1 *}$, Cassiano Rufino da Silva ${ }^{1}$, Emanuelle da Silva Ferreira ${ }^{2}$, Antonia do Nascimento ${ }^{1}$, Flávia Maria de França Alves ${ }^{1}$, Raíssa Luana Cardoso Sena ${ }^{1}$, Juan Carlos da Silva ${ }^{3}$, Guilherme de Andrade Ruela ${ }^{4}$, Andréa Rosane Sousa Silva ${ }^{1}$.

\section{RESUMO}

Objetivo: Revisar abordagens acerca da temática "educação em saúde para o público idoso", as quais podem incentivar e sensibilizar para a utilização de processos educativos com a população idosa em diversos contextos. Revisão bibliográfica: A saúde do idoso é um tema emergente e importante de ser discutido no âmbito social. E atualmente existem políticas públicas que podem garantir a qualidade de vida do idoso, mas, além disso, é necessário incluí-los de forma significativa na sociedade em que vivem e torná-los protagonistas do próprio movimento de envelhecimento saudável. Pois o processo de envelhecimento pode ser saudável e prazeroso, desde que haja mecanismos e propostas que viabilizem o cuidado para com a pessoa idosa. Tendo como alicerce a educação em saúde, é possível promover o autocuidado e elaborar atividades interativas e dinâmicas contextualizadas com a cultura da pessoa idosa. Considerações finais: A literatura mostra que, a saúde, como um campo multifatorial, enseja que esforços sejam realizados para tornar a educação em saúde cada vez mais difundida, compreendendo questões que ultrapassem os aspectos biológicos e também sociais, ambientais, culturais, entre outros. Para isso, é importante contar com ações que trabalhem a intersetorialidade e a inter/multi/transdisciplinaridade, considerando cada território e contexto.

Palavras-chave: Educação, Saúde, Envelhecimento.

\begin{abstract}
Objective: Review approaches on the theme of health education for the elderly, which can encourage and raise awareness for the use of educational processes with the elderly population in different contexts. Bibliographic review: The health of the elderly is an emerging and important topic to be discussed in the social sphere. And currently there are public policies that can guarantee the quality of life of the elderly, but, in addition, it is necessary to include them significantly in the society in which they live and make them protagonists of the healthy aging movement itself. Because the aging process can be healthy and pleasurable, as long as there are mechanisms and proposals that enable care for the elderly. Based on health education, it is possible to promote self-care and develop interactive and dynamic activities contextualized with the culture of the elderly. Final considerations: Literature shows that health, as a multifactorial field, requires efforts to be made to make health education more and more widespread, comprising issues that go beyond biological and also social, environmental, cultural aspects, among others. For this, it is important to have actions that work with intersectoriality and inter/multi/transdisciplinarity, considering each territory and context.
\end{abstract}

Key words: Education, Health, Aging.

\section{RESUMEN}

Objetivo: Revisar enfoques sobre el tema de la educación para la salud de las personas mayores, que puedan incentivar y sensibilizar para el uso de los procesos educativos con la población anciana en diferentes

\footnotetext{
${ }^{1}$ Centro Universitário Tiradentes de Pernambuco (UNIT-PE), Recife-PE. *E-mail: talita.flamin@gmail.com

2 Universidade Federal de Pernambuco (UFPE), Recife - PE.

${ }^{3}$ Centro Universitário Maurício de Nassau (UNINASSAU), Recife - PE.

${ }^{4}$ Universidade Federal de Juiz de Fora (UFJF), Juiz de fora - MG.
} 
contextos. Revisión de la literatura: La salud de las personas mayores es un tema emergente e importante para ser discutido en el ámbito social. Y actualmente existen políticas públicas que pueden garantizar la calidad de vida de las personas mayores, pero, además, es necesario incluirlas de forma significativa en la sociedad en la que viven y hacerlas protagonistas del propio movimiento de envejecimiento saludable. Porque el proceso de envejecimiento puede ser sano y placentero, siempre que existan mecanismos y propuestas que posibiliten el cuidado de las personas mayores. A partir de la educación para la salud, es posible promover el autocuidado y desarrollar actividades interactivas y dinámicas contextualizadas con la cultura de las personas mayores. Consideraciones finales: La literatura muestra que la salud, como campo multifactorial, requiere esfuerzos para que la educación en salud sea cada vez más generalizada, abarcando temas que van más allá de los aspectos biológicos y también sociales, ambientales, culturales, entre otros. Para ello, es importante contar con acciones que trabajen con intersectorialidad e inter / multi / transdisciplinariedad, considerando cada territorio y contexto.

Palabras clave: Educación, Salud, Envejecimiento.

\section{INTRODUÇÃO}

O envelhecimento populacional é um fenômeno mundial, sendo seus desdobramentos variáveis para cada sujeito. Estudos apontam que no Brasil tal fenômeno se intensificou a partir dos anos 70 (BORGES E, et al., 2017). Diante do contexto, é imprescindível a promoção de políticas públicas que visem bem-estar e a saúde para a população supracitada (FEITOSA EA, 2020).

Considerando a importância da saúde no âmbito da educação para a sociedade de modo geral, é relevante discutir as implicações desses aspectos no processo de envelhecimento humano. Assim sendo, torna-se necessário conhecer a história de vida, as preferências, as experiências e, sobretudo, quais são as atividades exercidas e os problemas que impactam na vida e na saúde do idoso. Nessa perspectiva, planos assistenciais e de cuidados, atividades e estratégias para promoção de saúde e (auto) cuidado são cruciais para esse público (SOUZA EM, et al., 2020).

De acordo com a Portaria no 2.528 de 19 de outubro de 2006, que aprova a Política Nacional de Saúde da Pessoa Idosa nas entrelinhas que perpassam o Sistema Único de Saúde (SUS), é indispensável a assistência integral e igualitária à saúde da pessoa idosa (BRASIL, 2006). Sendo essa de grande relevância para a efetivação dos direitos do idoso. Além disso, é importante frisar também que essa política visa a integração social efetiva e a autonomia do indivíduo, tendo como foco um dos principais problemas que comprometem a vida da pessoa idosa: a perda da capacidade física e mental no âmbito da realização das atividades do cotidiano (SANTOS MS, et al., 2018).

Ademais, é papel da família, da sociedade, do Estado e dos profissionais de saúde colaborar com a integralidade desse sujeito idoso (DIAS EG, 2018). Sendo imprescindível para este contexto considerar o conceito de saúde articulado a um viés holístico, conforme pressupõe a Organização Mundial da Saúde (OMS). Logo, de acordo com este órgão planetário, a saúde em sua amplitude, deve ser compreendida como uma conjunção de perfeito bem-estar físico, mental e social (WORLD HEALTH ORGANIZATION (WHO), 1948).

Deste modo, se torna fundamental considerar aspectos que interferem direta e indiretamente no bem-estar do sujeito, tais como: a pobreza, a violência, o saneamento básico, a moradia, dentre outros condicionantes saúde. Partindo dessa premissa, constatamos a necessidade do rompimento da visão biologicista que lamentavelmente ainda se arraiga. Esta perspectiva é historicamente conhecida como o Modelo Biomédico de atenção à saúde, no qual, o conceito de saúde reduz o processo saúde-doença à sua dimensão anatomofisiológica (MIRANDA SVC, et al., 2020).

De acordo com o evidenciado, mecanismos, contextos e fatores que refletem na saúde do idoso, precisam discutidos. Principalmente no que tange a esfera científica, pois é constatável que existem políticas públicas com o poder de viabilizar o processo de envelhecimento saudável, porém, há lacunas na efetivação destas políticas. Além do mais, nos últimos anos os serviços de assistência aos idosos estão aquém das necessidades apresentadas por estes. Podendo os sujeitos que compõem a sociedade em geral, buscar 
alternativas que minimizem os impactos negativos causados pela natureza do processo de envelhecer (ANDRADE LM, et al., 2013).

Considerando os supracitados, o presente artigo tem como foco a discussão sobre as relações entre educação em saúde e o processo de envelhecimento saudável. Outrossim, busca discutir sobre a necessidade dos hábitos e práticas saudáveis para a população idosa. Sendo a relevância deste estudo justificada por apresentar subsídios capazes de colaborar e/ou complementar os trabalhos atuais e futuros que discutem acerca da temática. Logo, o objetivo deste trabalho é revisar e divulgar informações e possíveis reflexões acerca da temática, as quais instigam a utilização de processos educativos envolvendo a população idosa em diversos contextos.

\section{REVISÃO BIBLIOGRÁFICA}

Os resultados que compreendem esta pesquisa estão agrupados e interconectados nos subtópicos abaixo, os quais estão caracterizados como categorias a partir da análise de conteúdo de Bardin (2016) com a finalidade de sistematizar as discussões e analisá-las. Logo, as categorias evidenciadas foram citadas como: "Afinal, o que é educação em saúde?", "A cultura, a educação popular e a pessoa idosa", e "Atividades de educação em saúde para o público idoso".

\section{Afinal, o que é educação em saúde?}

A educação é vista como um alicerce para o processo de desenvolvimento da sociedade. E quando é associada a fatores relacionados à saúde pode repercutir na progressão do bem-estar e da qualidade de vida. Por meio da educação em saúde é possível sistematizar metodologias, técnicas e estratégias que induzem o empoderamento, a autonomia e o autocuidado (OLIVEIRA RL e SANTOS MEA, 2011).

O Ministério da Saúde define educação em saúde como uma gama de práticas educativas direcionadas a população em geral, na perspectiva de estimular a autonomia e estabelecer uma comunicação horizontal entre a população, os profissionais, gestores e os diversos sujeitos envolvidos na promoção a saúde (MINISTÉRIO DA SAÚDE, 2008).

As práticas de educação em saúde envolvem três vertentes de atores prioritários: profissionais de saúde, que valorizam tanto a prevenção e promoção quanto as práticas de tratamento; gestores, que apoiam esses profissionais, contribuindo no planejamento de novos sistemas, ajudando assim, na modernização das informações; e pessoas que necessitam de conhecimento individual e coletivo, com maior autonomia para o cuidado (FALKENBERG MB, et al., 2014).

A educação em saúde é um campo multifacetado em que se conjugam diferentes conceitos dos dois âmbitos (educação e saúde) e que reflete diferentes compreensões do mundo a partir de distintas posições políticas e filosóficas da sociedade. A educação ampliada, inclui o reposicionamento de políticas públicas, e serviços de saúde adequados, além de sugestões pedagógicas libertadoras voltadas para o desenvolvimento da unidade e da cidadania, com foco na essência da melhoria da qualidade de vida e na promoção do ser humano (CARMO JB, 2014).

Salientando que, as práticas da educação em saúde são inerentes ao trabalho em saúde, mas, muitas vezes, está em segundo plano ou sequer é considerada no planejamento e organização dos serviços, na implementação e na gestão das ações (FALKENBERG MB, et al., 2014). Porém, na educação em saúde, a educação popular pode ser enfatizada, uma vez que essa contempla os conhecimentos prévios, culturais e a realidade em que se vive, e sobretudo, considerando como premissa os diversos saberes que compreendem a vida dos sujeitos e não apenas o conhecimento científico (FALKENBERG MB, et al., 2014).

Sem educação e os princípios teóricos e metodológicos que norteiam esse campo, a saúde em seu sentido amplo não pode ser alcançada, principalmente no âmbito da sua promoção. Talvez este seja o maior desafio para possibilitar esse tipo de diálogo, entender que todo esse processo requer envolvimento pessoal e um conhecimento mais profundo de sua trajetória de vida (PALÁCIO MAV e TAKENAMI I, 2020). 


\section{A cultura, a educação popular e a pessoa idosa}

Para uma visão ampla acerca da dinâmica de vida e do contexto social do idoso, é importante considerar diversas "singularidades", incluindo crenças, valores, hábitos e virtudes, as quais interferem de forma direta ou indireta na saúde dos sujeitos. Sendo assim, é cabível considerar a cultura como um elemento capaz de configurar o modo de vida das pessoas. Para Hall S (2016), a cultura é de definição complexa e envolve as ciências humanas e sociais, existem muitas maneiras de identificá-la. Na definição tradicional do termo, "cultura" é considerada como incluindo "as melhores coisas que foram consideradas e ditas" na sociedade. Além disso, pode-se utilizar a palavra "cultura" para se referir a música popular, publicações, arte, design e literatura ou atividades de lazer e entretenimento, o que está comum vida cotidiana das pessoas.

À vista disso, é possível afirmar que aspectos e elementos culturais perpassam a vida e o cotidiano dos indivíduos de forma geral. Isto porque o ser humano aprende de acordo com a cultura onde está inserido de forma natural e instantânea. E quando se trata da saúde, estes aspectos e elementos podem ser importantes para entender as experiências de adoecimento e as práticas de saúde defendidas pelo indivíduo e sua família, uma vez que os elementos culturais podem influenciar os hábitos e costumes e desordenar a conduta das pessoas em relação às demandas em saúde (MARTINS VV, 2013).

Se tratando da educação, que para Freire $\mathrm{P}$ (2019) pode ser entendida como um processo contínuo de aprendizado com base na realidade, capaz de repercutir nas tomadas de decisões críticas, reflexivas e conscientes, além de poder mudar o próprio eu e o mundo em que se vive. Sendo neste caso o indivíduo, o protagonista do seu próprio processo de aprendizado. Seguindo no âmbito da educação popular, Freire reitera a importância de uma maneira de construir uma educação baseada no conhecimento do popular, convidandoos a executar uma leitura da realidade na ótica do oprimido. Que neste caso, rompe as barreiras as quais se limitam a escrita e a leitura, considerando para além disso, as relações históricas e sociais (FREIRE P, 2019).

Levando em consideração os saberes do povo, os aspectos, pressupostos, ideias e elementos culturais para com o público idoso, é bastante comum suas associações aos processos históricos, considerando conceitos e valores passados de geração para geração. Além disso, o idoso apresenta características de contato com uma cultura, um espaço e uma temporalidade específica, sendo que tais pressupostos transcorrem a trajetória individual, social e cultural do sujeito (SILVA LC e FREITAS MCMA, 2018). Os idosos podem apresentar ideias e costumes próprios sobre o autocuidado, os quais permeiam alimentação, higiene, conforto, lazer e repouso. Frequentemente não existe preocupação com os aspectos científicos que envolvem a saúde, por considerarem os referidos hábitos e costumes sustentáveis e eficazes para suas necessidades diversas (FALLER JW, MARCON SS, 2013).

No entanto, é notável que, os elementos, ideias e perspectivas desencadeadas pela ciência, podem viabilizar e colaborar com a promoção de práticas de vida saudáveis que acentuem o bem-estar. Ainda assim, os saberes culturais e populares também podem ser potentes para o desenvolvimento pleno de cidadania e de hábitos de vida saudáveis, de modo amplo. Para Manhães FC e Souza CHM (2018), a conscientização e a ressignificação de hábitos de vida configuram que o bem-estar é essencial para o que se entende como envelhecimento saudável. Assim, entende-se que é importante oportunizar um amplo diálogo em busca da ideação do conhecimento sobre saúde. Isso pode se dar através da troca de experiências e saberes, repercutindo assim, na motivação necessária para garantir o autocuidado ao longo do tempo (MEURER ST, et al., 2012).

\section{Atividades de educação em saúde para o público idoso}

Quando se fala em atividades educativas para a população idosa é importante considerar as limitações funcionais, cognitivas e anatomofisiológicas dos sujeitos. Nesse sentido, é apontado o entendimento de Ferreira OGL, et al. (2012) sobre esse processo do envelhecimento que leva a um declínio gradual da capacidade funcional, e esse declínio aumentará gradativamente com a idade. Portanto, a maior adversidade à saúde relacionada ao envelhecimento é a disfunção e dependência, que pode levar à limitação / perda de habilidades ou dificuldade / incapacidade para desempenhar funções e atividades relacionadas à vida diária. Essas dificuldades são causadas por limitações físicas e cognitivas, de forma que a saúde da população idosa 
pode ser determinada por diversos indicadores específicos, incluindo a presença de déficits físicos e cognitivos.

Logo, as dificuldades ocasionadas pelas limitações físicas e cognitivas são evidentes e a educação em saúde pode trazer para o sujeito idoso melhorias significativas em sua vida. Porém, considerando, no âmbito das práticas e dinâmicas para esse público, deve-se levar em consideração o bom e o mau uso do corpo, propondo-lhes atividades adequadas para cada circunstância que esteja passando. A fim de que, os mecanismos e exercícios propostos atinjam os mais diversos objetivos de saúde. Pois, a prática irregular de determinadas atividades físicas, como por exemplo o exagero de exercícios físicos, pode causar danos irreparáveis a saúde do indivíduo (SANTOS AA, 2019).

É importante estimular o idoso, por meio da educação em saúde, a buscar realizar atividades práticas prazerosas, seja de forma individual ou em grupo, uma vez que tais propostas são capazes de mantê-lo ativo fisicamente e psicologicamente. Portanto, elenca-se atividades como jogo de xadrez, quebra-cabeça, palavras cruzadas, baralho e dominó como jogos educativos que podem ajudar a estimular o raciocínio lógico, a manutenção da memória. Sendo os jogos caracterizados como instrumentos de intervenções psicopedagógicas, uma vez que para se jogar geralmente é necessário estimular pensamentos específicos para cada jogada e ação (SANTOS ACP, 2016).

As atividades mencionadas podem ser realizadas de forma gratuita ou com muito pouco recurso financeiro. Sobre esse aspecto também já foi considerado que geralmente, na velhice, os problemas de saúde causados por múltiplas patologias são agravados pela solidão e pela pobreza. A inatividade e a falta de perspectivas de aposentadoria podem levar a sentimentos de depressão, o que pode prejudicar a saúde do indivíduo. Pois o idoso tem de ter segurança para lidar com os problemas de saúde, e apresentar alta autoestima, sendo essa última uma grandiosa influenciadora no cotidiano do sujeito idoso (ROCHA ERM, 2017).

Além disso, os pressupostos teóricos e práticos são indissociáveis, principalmente na esfera da aprendizagem educacional, quando se considera o ensino de qualidade. Isto quer dizer que o que se discute e o que se faz estão intrinsecamente interligados, ou seja, não podem ser separados. Nesse sentido, entendese que é necessário que ocorra a mobilização de saberes, isto quer dizer que os conhecimentos teóricos construídos devem ser praticados no cotidiano, seja através de uma atividade educativa de natureza mental, de uma atividade física, e/ou até mesmo através de uma atividade de autocuidado (SILVA TSG, 2019).

Por outro lado, práticas educativas que estimulem exercícios de caráter físico/prático, tais como a hidroginástica, a dança e a ginástica aeróbica, podem estimular a circulação sanguínea, prevenir problemas respiratórios e cardiovasculares, além de ajudar no desempenho das articulações dos sujeitos. Com base nisso, é cabível enfatizar que as práticas de educação em saúde podem atuar de forma efetiva e potencializadoras no processo natural de envelhecimento, principalmente no âmbito da prevenção de doenças (MINISTÉRIO DA SAÚDE, 2006).

Nessa perspectiva, vale ressaltar a importância de planejar ações e atividades que promovam a saúde e previnam agravamentos recorrentes do processo do envelhecimento. Pois dessa maneira, é possível manter a capacidade funcional do idoso, fazendo-o perceber como uma pessoa mais ativa. Além disso, é de extrema relevância apontar, de forma breve, que planejar uma proposta educativa em saúde vai muito mais além de apenas sistematizar, pois para isso é importante considerar as necessidades e a realidade do sujeito envolvido (BRASIL, 2006).

\section{CONSIDERAÇÕES FINAIS}

O processo de envelhecimento é contínuo, complexo e com especificidades. É de extrema importância que os profissionais que atuam nas múltiplas esferas sociais se apropriem de saberes, competências e habilidades pautadas em evidências científicas e as agreguem à prática laboral voltada aos idosos, em especial em práticas educativas para a promoção a saúde. A literatura mostra que, a saúde, como um campo multifatorial, enseja que esforços sejam realizados para tornar a educação em saúde cada vez mais difundida, compreendendo questões que ultrapassem os aspectos biológicos e também sociais, ambientais, culturais, 
entre outros. Para isso, é importante contar com ações que trabalhem a intersetorialidade e a inter/multi/transdisciplinaridade, considerando cada território e contexto dos indivíduos nesse ciclo vital para um envelhecimento ativo.

\section{REFERÊNCIAS}

1. ANDRADE LM, et al. Políticas públicas para pessoas idosas no Brasil: uma revisão integrativa. Ciência \& Saúde Coletiva. 2013; 18(12): 3543-52

2. BARDIN L. Análise de conteúdo. São Paulo: Edições 70, 2016; 279p.

3. BORGES E, et al. O envelhecimento populacional: um fenômeno mundial. O envelhecimento populacional: um fenômeno mundial. Aspectos biopsicossociais do envelhecimento e a prevenção de quedas na terceira idade. Joaçaba: Editora Unoesc, 2017.

4. BRẢSIL. Portaria no 2.528, de 19 de outubro de 2006. Aprova a Política Nacional de Saúde da Pessoa Idosa. 2006. Disponível em: https://bvsms.saude.gov.br/bvs/saudelegis/gm/2006/prt2528_19_10_2006.html. Acesso em: 8 ago. 2021.

5. CARMO JB. Educação em saúde: implementação das práticas educativas no contexto da HAS. Monografia (Especialização em Linhas de Cuidado em Enfermagem - Doenças Crônicas Não Transmissíveis) - Departamento de Enfermagem, Universidade Federal de Santa Catarina, Florianópolis, 2014.

6. CERVO AL, BERVIAN PA. Metodologia científica. São Paulo: Makron Books, 1996; p. 209.

7. DIAS EG. Adesão de idosos aos tratamentos da hipertensão arterial e as boas práticas de cuidado na perspectiva da integralidade. Dissertação (Mestrado em Tecnologia e Inovação em Enfermagem) - Escola de Enfermagem de Ribeirão Preto, Universidade de São Paulo, Ribeirão Preto, 2018.

8. FALKENBERG MB, et al. Educação em saúde e educação na saúde: conceitos e implicações para a saúde coletiva. Ciência \& Saúde Coletiva, 2014; 19: 847-852.

9. FALLER JW, MARCON SS. Práticas socioculturais e de cuidado à saúde de idosos em diferentes etnias. Esc Anna Nery, 2013; 17(3): 512-519.

10. FEITOSA EA. As Legislações Brasileiras São Instrumentos que Garantem a Autoestima do Idoso?. Revista Gestão \& Políticas Públicas, 2020; 10(2): 278-290.

11. FERREIRA OGL, et al. Envelhecimento Ativo e Sua Relação Com a Independência Funcional. Texto e Context Enferm. 2012; $21(3):$ 513-8.

12. FREIRE P. Pedagogia da autonomia: saberes necessários à prática educativa. $68^{\mathrm{a}}$ ed. Rio de Janeiro: Paz e Terra, 2019.

13. HALL S. Cultura e representação. Rio de Janeiro: PUC-Rio; Apicuri, 2016; 259 p.

14. MANHÃES FC, SOUZA CHM. Envelhecimento humano em processo. Brasil Multicultural, 2018; $376 \mathrm{p}$.

15. MARTINS VV. Saúde sexual de mulheres com estomia na perspectiva da teoria de Nola Pender. Dissertação (Mestrado em Enfermagem) - Centro Biomédico, Universidade do Estado Rio de Janeiro, Rio de Janeiro, 2013.

16. MEURER ST, et al. Fatores motivacionais de idosos praticantes de exercícios físicos: um estudo baseado na teoria da autodeterminação. Estudos de Psicologia, 2012; 17(2): 299-303.

17. MINISTÉRIO DA SAÚDE. Secretaria de Atenção à Saúde. Departamento de Atenção Básica. Envelhecimento e saúde da pessoa idosa. Cadernos de Atenção Básica. 2006. Disponível em:

https://aps.saude.gov.br/biblioteca/visualizar/MTE5NQ==. Acesso em: 8 ago. 2021.
18. MINISTERIO DA SAÚDE. Tesauro Eletrônico. Educação em Saúde. 2008. Disponível em: 2021.

19. MIRANDA SVC, et al. A visão do homem trabalhador rural norte-mineiro sobre o cuidado em saúde no contexto da atenção primária à saúde. Ciência \& Saúde Coletiva, 2020; 25(4):1519-1527.

20. OLIVEIRA RL, SANTOS MEA. Educação Em Saúde Na Estratégia Saúde Da Família: Conhecimentos E Práticas do Enfermeiro. Revista Enfermagem Integrada, 2011; 4(2).

21. PALÁCIO MAV, TAKENAMI I. Em tempos de pandemia pela COVID-19: o desafio para a educação em saúde. Vigilância Sanitária em Debate: Sociedade, Ciência \& Tecnologia (Health Surveillance under Debate: Society, Science \& Technology) - Visa em Debate, 2020, 8(2): 10-15.

22. ROCHA ERM. Relações sócio afectivas, envelhecimento ativo e bem-estar das pessoas mais velhas. Dissertação (Mestrado em Psicogerontologia Comunitária) - Escola Superior de Educação, Instituto Politécnico de Beja, Beja, 2017.

23. SANṪOS AA. Avaliação das dores musculares e articulares em praticantes de exercícios físicos em academias e suas correlações. Monografia (Graduação em Fisioterapia) - Centro Universitário de Lavras, Lavras, 2019.

24. SANTOS MS, et al. Práticas integrativas e complementares: Avanços e desafios para a promoção da saúde de idosos. Revista Mineira de Enfermagem., 2018; 22(1126).

25. SANTOS ACP. Jogos de mesa na terceira idade e sua importância para a Aprendizagem: um olhar psicopedagógico. Trabalho de Conclusão de Curso (Bacharelado em Psicopedagogia) - Universidade Federal da Paraíba, João Pessoa, 2016.

26. SILVA TSG. Ensino de ciências e experimentação nos anos iniciais: da teoria à prática. Pró-Discente, 2019; 25(1): 41-53.

27. SILVA LC, FREITAS MCMA. Recontando histórias e revivendo memórias: A contação de histórias como resgate de memória para idosos. Revista Educação, Ciência e Inovação. 2018; 3(1):121-31.

28. SOUZA EM, et al. Educação popular, promoção da saúde e envelhecimento ativo: uma revisão bibliográfica integrativa. Ciência \& Saúde Coletiva, 2021;26: 1355-1368.

29. WORLD HEALTH ORGANIZATION (WHO). Declaração Universal dos Direitos Humanos: Constituição da organização Mundial da Saúde, $1948 . \quad$ Disponível em: http://www.direitoshumanos.usp.br/index.php/OMSOrganiza\%Ċ\%A7\%C3\%A30-MundialdaSa\%C3\%BAde/constituicao-da-organizacaomundial-da-saude-omswho.html. Acesso em: 18 out. 2021. 